\title{
A NUMERICAL STUDY OF THE SOLAR CYCLE
}

\author{
Y. NAKAGAWA \\ High Altitude Observatory, National Center for Atmospheric Research*, Boulder, Colo., U.S.A.
}

\begin{abstract}
Models relevant to numerical studies of the solar cycle are reviewed briefly with discussions of pertinent physical mechanisms. It is suggested that the observed surface activities are secondary in nature and an example of possible non-axisymmetric steady state solutions is given, together with the results of preliminary numerical computations.
\end{abstract}

\section{Introduction}

In the model study of the solar cycle, it is necessary that a model satisfies various well-established laws of observations. These laws are: (1) Hale's law of polarity of sunspots (Hale, 1908, 1913), namely, the preceding sunspot has opposite polarity in the northern and southern hemispheres and their polarities reverse from one 11-yr cycle to the next, (2) Spörer's law of zone of activity (Carrington, 1858; Spörer, 1894), i.e., at the beginning of activity the sunspots appear near latitude $30^{\circ}$ and the zone of activity gradually shifts towards the equator in $11 \mathrm{yr}$, and (3) the reversal of polar magnetic field (Babcock, 1959) with approximately 11-yr period.

Models which satisfy some or all of these laws of observations have been proposed by a number of authors on the basis of somewhat diversified physical mechanisms. Among the models, notably, the topological model of Babcock (1961) has combined the observed features of the 22-yr cycle of the Sun in a consistent manner, and most other models have been based on this topological model in one way or the other. The first quantitative examination of the Babcock model (1961) has been given by Leighton (1964). Leighton (1964) showed that the redistribution and annihilation of magnetic flux of bipolar sunspots by supergranulation could account for the development of the bipolar magnetic regions which lead to the final reversal of the polarity of polar magnetic field.

A more extended quantitative examination of the Babcock model has been presented by Nakagawa and Swarztrauber (1969). Starting with the initial state of axisymmetric quasi-dipole magnetic field they have numerically integrated the hydromagnetic equations within the context of axisymmetric incompressible hydromagnetics. Their results showed in agreement with the Babcock model, (1) the development of the toroidal magnetic field, (2) the gradual drift of zone of activity (Spörer's law), and (3) Hale's law of polarity. However, the assumption of axisymmetry limited their study only to a single 11-yr cycle. Recently a different type of approach to the model of the solar cycle has been suggested by Leighton (1969), in the form of a 'magneto-kinematic' model based on a set of synthesized equations which govern the magnetic fields. It was mentioned that these equations account for the various physical processes needed at

* The National Center for Atmospheric Research is sponsored by the National Science Foundation 
the different stages of development of the solar cycle as postulated in the Babcock model. However, it is rather difficult to justify his basic equations from the ordinary hydromagnetics.

Apart from these studies, in a series of papers, Krause (1967) and his collaborators have developed a model of the solar cycle, emphasizing the effect of hydromagnetic turbulence resulting from the subphotospheric convection. Their model utilized an induced quadrupole type magnetic field first developed near the surface which migrates towards the equator to account for Spörer's law of zone of activities and towards the interior to achieve the polarity reversal of initial dipole-like polar magnetic field.

In this paper, the basic concept of the model of an the solar cycle under the assumption of non-axisymmetric hydromagnetics is discussed and some preliminary results of a non-axisymmetric hydromagnetic model study of an incompressible, viscous medium of finite electrical conductivity are presented.

\section{Basic Concept of the Model}

In constructing a model of the solar cycle, the question must be focused on the physical mechanisms which are pertinent to produce the observed phenomena. First, we may follow the Babcock model (1961) to examine the various physical mechanisms postulated in his model. Babcock (1961) divided his model into five successive stages of development each characterized by the dominance of different physical mechanisms.

The initial stage (stage 1) corresponding to the state of solar minimum was assumed to be dominated by an approximately axisymmetric dipole-like magnetic field with the interior flux lying in a relatively thin submerged layer, while the surfaces of constant angular velocity (isotachs) cut more deeply into the Sun than the magnetic lines of force. This assumption led to stage 2 of the generation and amplification of toroidal magnetic field from the initial poloidal magnetic field by the differential rotation. This was followed by stage 3 , the emergence of the amplified toroidal magnetic field as bi-polar sunspots due to a postulated instability associated with the magnetic buoyancy. Stage 4 was characterized by the redistribution and annihilation of the emerged magnetic flux which eventually led to the reversal of polarity of the polar magnetic field. The final stage 5 was assumed to be the solar minimum reached after 11-yr similar to stage 1 but with the dipole polarity reversed.

In Leighton's model (Leighton, 1964), the redistribution and cancellation of bipolar magnetic flux (the stage 4 of Babcock model) was shown to be the result of a twodimensional random walk process due to the supergranulation in the presence of a differential rotation. Leighton (1964) utilized an important result of observation that the bipolar sunspots emerge with a slight difference in the latitude governed by Hale's law, i.e., the preceding spot appears closer to the equator. The use of this result led the polarity of reversed polar magnetic field to be that of the following spot.

In the hydromagnetic model of Nakagawa and Swarztrauber (1969), the law of isorotation (Ferraro, 1937) was assumed at the initial stage. As a consequence, the angular velocity of their model decreased with depth. However, in the presence of 
viscous dissipations (which could be identified with the granulation as well as supergranulation), a net angular velocity transfer towards the equator was required to maintain the faster equatorial rotation. The angular momentum transfer was achieved by a meridional circulation directed towards the equator near the surface. This meridional circulation produced a concentration of the interior magnetic lines of force near the surface similar to the Babcock model of stage 1, and led to the subsequent generation and amplification of toroidal magnetic field of stage 2. The manner of distortion of the initial poloidal magnetic lines of force then successfully accounted for Hale's law. Spörer's law of the equatorial migration of the zone of activity was satisfied by the gradual equatorial drift of the concentrated toroidal magnetic field by meridional circulation. In contrast to this model, Krause (1967) and Leighton (1969) have assumed a differential rotation increasing with depth.

The initial state of Krause's model (Krause, 1967) was characterized by a prevailing dipole-like magnetic field which cut deeply into the bottom of the convection zone contrary to the shallow submergence assumed by Babcock (1961). Also, as stated above, it was assumed that the core rotates faster than the outer surface in his model. The assumed differential rotation induced a toroidal magnetic field similar to stage 2 of the Babcock model, however, the hydromagnetic turbulence coupled with the density stratification led to the production of quadrupole type poloidal magnetic field with the polarity opposite to the initial dipole-like field. The subsequent emergence of the quadrupole type magnetic field was identified as the solar activity, and the final polarity reversal of the initial dipole-like magnetic field was achieved by a gradual migration of the induced quadrupole type magnetic field towards the interior.

Apart from the difficulties of justification of the basic equations, the 'magnetokinematic' model by Leighton (1969) has been most successful, as this model has reproduced the observed solar cycle over three 22-yr cycles including fluctuations. Therefore, it may be worthwhile to summarize some of the assumptions and conclusions relevant to the present discussions. Those are: (1) a faster interior rotation is preferred, (2) the differential rotation confined close to the equator is also preferred, (3) the formation of bipolar sunspots can be considered as a random (eruption) process, (4) the emerged bipolar sunspots have the latitudinal difference given by Hale's law, and (5) the redistribution and annihilation of erupted magnetic flux, including the reversal of polarity of the polar magnetic field, are the result of a two-dimensional random walk process due to supergranulation in the presence of a differential rotation.

In view of the quasi-regularity of the solar cycle, we may consider that the observed surface phenomena could be perturbations (finite amplitude or otherwise) over a possible, long-time averaged, basic state of approximately axisymmetric equilibrium. Then, it may be worthwhile to examine the physical characteristics of the state of axisymmetric hydromagnetic equilibria. It has been shown (Woltjer, 1959a, b; Mestel, 1961; Nakagawa, 1970b) that for a perfectly conducting medium, the meridional velocity field must be similar to the poloidal magnetic field. Hence, for a convective circulation confined within a spherical shell, the poloidal magnetic field must also be confined within the same shell. Further, it has been shown that if the boundary of 
the shell is formed by a line of force of the poloidal magnetic field, the velocity of rotation must be a constant. Table I presents a summary of the principal characteristics of axisymmetric equilibria deduced from the integrals of hydromagnetic equations. It can be seen from Table I that with a meridional circulation the possible surface rotation is at most a constant. In this connection, it is interesting to note that Wilcox (1971) found that the large scale magnetic pattern of the Sun rotates with practically a constant angular velocity of rotation, while the movement of sunspots and the Doppler shift observations showed the faster equatorial differential rotation.

\section{TABLE I}

Summary of principal physical characteristics of axisymmetric hydromagnetic equilibria

$\begin{array}{ll}\text { Case } A & \text { I } \text { Constant surface rotation } \\ & \text { II No surface rotation } \\ & \text { III No rotation (anywhere) }\end{array}$

Case $B \quad$ I $\quad$ Constant surface rotation

II Surface differential rotation possible

III Surface differential rotation possible with magnetic field extending outside

Case $A$ meridional circulation, Case $B$ no meridional circulation, Subcase $I$ poloidal and toroidal magnetic field, Subcase II purely toroidal magnetic field, Subcase III purely poloidal magnetic field.

In view of this conclusion, it appears logical to consider that the observed differential rotation of the Sun could be a locally confined phenomenon (Leighton, 1969). Also, it appears reasonable to assume that the general surface magnetic fields are the results of leakages or eruptions of interior magnetic fields as postulated by Babcock (1961) and Leighton (1969) rather than a systematic large scale field. Then we may consider that the first task of the hydromagnetic model study should be directed to the determination of an interior self-consistent systematic large scale magnetic field and convective flow.

\section{Basic Equations and Boundary Conditions}

The basic equations of the problem could be divided into two groups, i.e., the equations governing the hydromagnetics and the equations governing the thermodynamics of the problem. In the present study, we consider only the hydromagnetics of the problem and in particular, an incompressible medium so that the successive future improvements of the model such as the introduction of compressibility of medium and thermodynamics of the problem would provide us with the understanding of the importance of these effects.

For an incompressible medium, the appropriate equations of hydromagnetics can be written in the following forms, in a system of coordinates rotating with a constant 
velocity $\Omega$ around the polar axis:

$$
\begin{aligned}
\frac{\partial \mathbf{v}}{\partial t}= & (\boldsymbol{\nabla} \times \mathbf{B}) \times \mathbf{B}-(\boldsymbol{\nabla} \times \mathbf{v}) \times \mathbf{v}-\boldsymbol{\nabla}\left(\frac{1}{2}|\mathbf{v}|^{2}\right)- \\
& -2 \mathbf{\Omega} \times \mathbf{v}-\boldsymbol{\nabla} \Pi-v \boldsymbol{\nabla} \times(\boldsymbol{\nabla} \times \mathbf{v}), \\
\frac{\partial \mathbf{B}}{\partial t}= & \boldsymbol{\nabla} \times(\mathbf{v} \times \mathbf{B})-\eta \boldsymbol{\nabla} \times(\boldsymbol{\nabla} \times \mathbf{B}), \\
\boldsymbol{\nabla} \cdot \mathbf{v}= & 0, \\
\boldsymbol{\nabla} \cdot \mathbf{B}= & 0,
\end{aligned}
$$

where $\mathbf{v}$ is the velocity, $\mathbf{B}$ the magnetic field measured in terms of velocity unit $\left[\mathbf{B}=\mathbf{B}^{*}(4 \pi \varrho)^{-1 / 2} ; \mathbf{B}^{*}\right.$ the strength of magnetic field induction, $\varrho$ the density], $\Pi$ a scalar defined by $\Pi=p \varrho^{-1}-\frac{1}{2} \tilde{\omega}^{2}|\Omega|^{2}+\Phi(p$ the pressure, $\tilde{\omega}$ the radial distance from the rotating axis, $\Phi$ the gravitational potential), $v$ the kinematic viscosity, and $\eta$ the magnetic diffusivity. In deriving Equations (1) and (2), $v$ and $\eta$ are assumed constants.

The basic equations of the present numerical study consist of a set of seven equations: three components of Equation (1), three components of Equation (2) and an equation for $\Pi$ which is obtained by taking the divergence of Equation (1), i.e.,

where

$$
\nabla^{2} \Pi=\nabla[(\nabla \times \mathbf{B}) \times \mathbf{B}]-\nabla[(\boldsymbol{\nabla} \times \mathbf{v}) \times \mathbf{v}]-\nabla^{2}\left(\frac{1}{2}|\mathbf{v}|^{2}\right)-2 \nabla(\mathbf{\Omega} \times \mathbf{v}),
$$

$$
\nabla^{2}=\begin{array}{cc}
1 & \partial \\
r^{2} & \partial r
\end{array}\left(r^{2} \frac{\partial}{\partial r}\right)+\frac{1}{r^{2} \sin \theta} \frac{\partial}{\partial \theta}\left(\sin \theta \frac{\partial}{\partial \theta}\right)+\frac{1}{r^{2} \sin ^{2} \theta \partial \phi^{2}}, \partial^{2},
$$

and $r, \theta, \phi$, denote the radius, co-latitude and longitude, respectively. These equations are currently solved numerically subject to the boundary conditions described below.

In the model, the convection zone of the Sun is represented by a spherical shell between radius $r=1$ and $r=\beta(\beta<1)$. Then, in order to simulate the effect of convection, which could be interpreted in terms of the differential rotation (Kato and Nakagawa, 1969; Busse, 1970), we shall assume that: (1) The observed differential rotation is maintained at the outer surface $(r=1)$; $(2)$ at the bottom (the core) surface $(r=\beta)$, a constant rotation is maintained; (3) the fluid motions are confined within the spherical shell and (4) the magnetic fields are also confined within the spherical shell.

The last condition is assumed in consideration of the physical characteristics of the averaged state of axisymmetric equilibria. In other words, the present study is focused on the investigation of the possible recycling of the subsurface flow and magnetic field consistent with the basic hydromagnetic equations subject to the boundary conditions representing the averaged state.

At the beginning of our computation, we select an arbitrary set of approximate steady state non-axisymmetric solutions in order to facilitate the study. The choice of such solutions is designed to introduce certain longitudinal perturbations which otherwise cannot be generated from the equations. The possible choice of a set of non-axisymmetric solutions follows from the fact that a divergence free vector can be 
represented by two scalar functions, say, $P(r, \theta, \phi)$ and $T(r, \theta, \phi)$. For example, we can express the velocity field by (e.g. Chandrasekhar, 1961),

$$
\begin{aligned}
\mathbf{v}=\boldsymbol{\nabla} \times \boldsymbol{\nabla} \times\left(P \mathbf{1}_{r}\right) & +\nabla \times\left(T \mathbf{1}_{r}\right)= \\
=\left[-\frac{\Lambda^{2}}{r^{2}} \boldsymbol{P}\right] \mathbf{1}_{r} & +\left[\frac{1}{r} \frac{\partial^{2} P}{\partial r \partial \theta}+\frac{1}{r \sin \theta} \frac{\partial T}{\partial \phi}\right] \mathbf{1}_{\theta}+ \\
+ & {\left[\frac{1}{r \sin \theta} \frac{\partial^{2} P}{\partial r \partial \phi}-\frac{1}{r} \frac{\partial T}{\partial \theta}\right] \mathbf{1}_{\phi} }
\end{aligned}
$$

where

$$
\Lambda^{2}=\frac{1}{\sin \theta} \frac{\partial}{\partial \theta}\left(\sin \theta \frac{\partial}{\partial \theta}\right)+\frac{1}{\sin ^{2} \theta} \frac{\partial^{2}}{\partial \phi^{2}}
$$

and $\mathbf{1}_{r}, \mathbf{1}_{\theta}$, and $\mathbf{1}_{\phi}$ denote unit vectors in the three principal directions.

In the present problem, we may write the scalar functions in terms of spherical surface harmonics,

$$
Y_{l}^{m}(\theta, \phi)=P_{l}^{|m|}(\cos \theta) e^{i m \phi},
$$

where $P_{l}^{m}(\cos \theta)$ is the Legendre function (e.g. Janke and Emde, 1945). Introducing the notations $U, V$, and $W$ for the radial, latitudinal, and longitudinal velocity components, and choosing

$$
P={ }^{(I)} \Phi(r){ }^{(I)} Y_{l}^{m}(\theta, \phi), \quad T={ }^{(I I)} \Phi(r){ }^{(I I)} Y_{l}^{m}(\theta, \phi),
$$

we obtain

$$
\begin{aligned}
& U=\frac{1}{r^{2}}{ }^{(I)} \Phi(r) l(l+1){ }^{(I)} Y_{l}^{m}(\theta, \phi), \\
& V=\frac{1 \mathrm{~d}}{r \mathrm{~d} r}{ }^{(I)} \Phi(r){ }_{\partial \theta}^{\partial{ }^{(I)}} Y_{l}^{m}(\theta, \phi)+{ }_{r}^{1}{ }^{(I I)} \Phi(r) \frac{1}{\sin \theta} \partial \phi{ }^{(I I)} Y_{l}^{m}(\theta, \phi), \\
& W=\begin{array}{l}
1 \mathrm{~d} \\
r \mathrm{~d} r
\end{array}{ }^{(I)} \Phi(r) \begin{array}{c}
1 \\
\sin \theta \partial \phi
\end{array}{ }^{(I)} Y_{l}^{m}(\theta, \phi)-{ }_{r}^{1}{ }^{(I I)} \Phi(r){ }_{\partial \theta}^{\partial}{ }^{(I I)} Y_{l}^{m}(\theta, \phi),
\end{aligned}
$$

where we have used the relation

$$
\Lambda^{2} Y_{l}^{m}(\theta, \phi)=-l(l+1) Y_{l}^{m}(\theta, \phi)
$$

It should be noted that the components of magnetic field can be written in similar forms regardless of the assumption concerning compressibility of the medium, and that for a compressible medium the components of velocity at a steady state can be given by replacing $U, V$, and $W$ with $\varrho U, \varrho V$, and $\varrho W$, respectively.

If we assume further that the flow has symmetry with respect to the polar axis $(\theta=0)$ and the equatorial plane $(\theta=\pi / 2)$, we find the following conditions for the choice of $l$ and $|m|$, excluding $|m| \neq 0$,

$$
\begin{aligned}
& l \geqslant|m|, \quad|m| \geqslant 2 \\
& l-|m|= \begin{cases}0 & \text { or even integers for }(I) \\
\text { odd integers for }(I I) .\end{cases}
\end{aligned}
$$


An example of such a solution in the presence of a strong axisymmetric toroidal magnetic field is shown in Figure 1. The unipolar regions in Figure 1 are identified with the variations of the magnetic field near the surface, so that the flux coil which appeared near the surface is identified as one polarity, while the submerged region is the opposite polarity. It may be noted that in the absence of a surface differential rotation we may choose $\mathbf{v} \| \mathbf{B}$, so that the magnetic flux could be considered to represent the flow fields. Also it should be noted that without a strong axisymmetric toroidal magnetic field the solutions with the choise of $l$ and $m$ given in the condition (15) represent celluar structures rather than the continuous configurations shown in Figure 1.

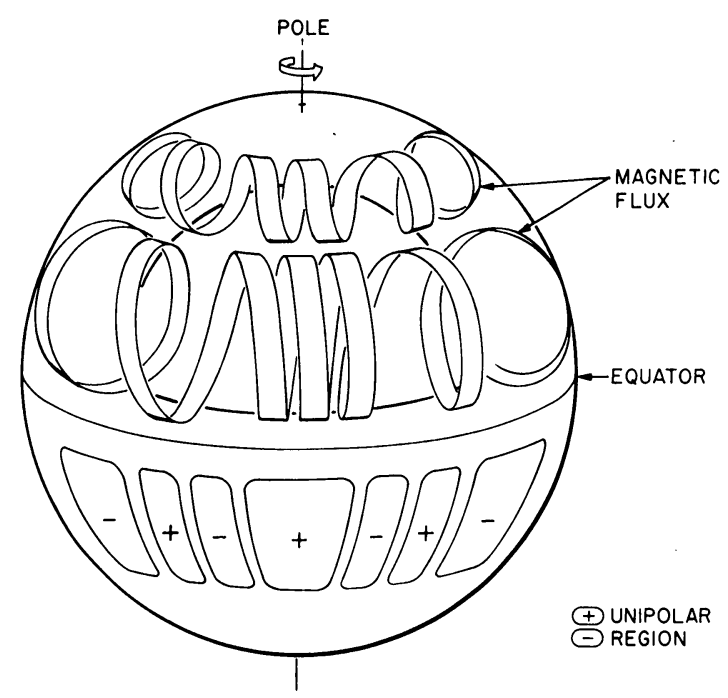

Fig. 1. An example of schematic large scale non-axisymmetric magnetic field.

In the present problem, however, with the assumed surface differential rotation, we have $W(\theta) \neq 0$ at $r=1$ while $\mathbf{B}=0$ at $r=1$. Hence, $\mathbf{v} \# \mathbf{B}$ near the surface (also in the interior), this boundary condition leads to the generation of an induced magnetic field by the flow near the surface. The subsequent interactions between the flow and magnetic fields then lead to the non-linear variations (oscillations) of the whole flow and magnetic field. It is considered that these non-linear oscillations represent the solar cycle in our model.

\section{Results and Discussions}

The results which remain somewhat preliminary at present indicate that the velocity field distorted strongly near the surface (to satisfy the surface boundary condition of the observed differential rotation) induces a strong distortion of magnetic fields and subsequently oscillations of both velocity and magnetic fields. The strong distortion of magnetic fields near the surface could be considered as the zone of activity. It is 
found that the oscillations of the flow and magnetic fields propagate in both the longitudinal and latitudinal directions, somewhat similar to the results of linear analyses of the Rossby-type waves by Kato (1969), and Kato and Nakagawa (1970). The propagation of such waves could be identified with the change of pitch of the magnetic flux shown in Figure 1, thus the migration of large unipolar regions. From the surface differential rotation given by Newton and Nunn (1951) and the differential rotation monotonically decreasing with depth we find that the zone of activity gradually migrates towards the equator, while each unipolar region grows and decays in the order of a year.

However, more detailed studies are needed since a number of parameters involved require further examination. For example, with faster interior rotation, we may still satisfy Spörer's law of zone of activity by increasing the number of meridional circulation cells so that near the surface the circulation is directed toward the equator. Further, it should be noted that the strength of the interior magnetic field could be taken somewhat arbitrary irrespective of the emerged strength of the sunspot magnetic field. The requirement of the virial theorem (e.g. Nakagawa and Trehan, 1970) could be satisfied by an interior magnetic field of strength well over $10^{6} \mathrm{G}$.

In the analysis by Kato and Nakagawa (1970), it was shown that the most unstable waves propagate with a sub-Alfvénic speed, and the present preliminary results support this prediction. In this connection, it may be worthwhile to note that in the state of axisymmetric equilibria, it has been shown (Mestel, 1961; Nakagawa, 1970b) that the poloidal convective velocity must remain either super-Alfvénic or sub-Alfvénic throughout the circulation. This condition may provide the limit to the strength of the interior magnetic field, if a realistic velocity of the interior convective circulation is determined.

It is advantageous to start our discussions with the results of previous axisymmetric incompressible study (Nakagawa and Swarztrauber, 1969), as the basic physical mechanisms of the problem are represented in the model. Figure 2 shows the developments of the toroidal magnetic field in the axisymmetric model by differential rotation at $2,4,6,8,10$, and $12 \mathrm{yr}$ after the initial state, when the surface differential rotation is given by the formula of Newton and Nunn (1951). The migration of a strong toroidal magnetic field developed near the surface towards the equator can be seen clearly in Figure 2. Another representation of this migration of the toroidal magnetic field in the form of the 'Butterfly' diagram is shown in Figure 3. It should be noted that the results of the study also showed that the poleward migration of active regions (identified with the maxima of the induced toroidal magnetic field) results with the formula of the surface differential rotation given by d'Azambuja (1948).

We may now discuss the similarities and differences of the present non-axisymmetric model and the previous axisymmetric model. In both models, the viscous dissipation due to granulation and supergranulation are considered, thus a net angular velocity transfer towards the equator is required, in order to maintain the faster equatorial rotation. This angular momentum transfer was achieved by a meridional circulation directed towards the equator near the surface. Also in both models, the zone of activity 

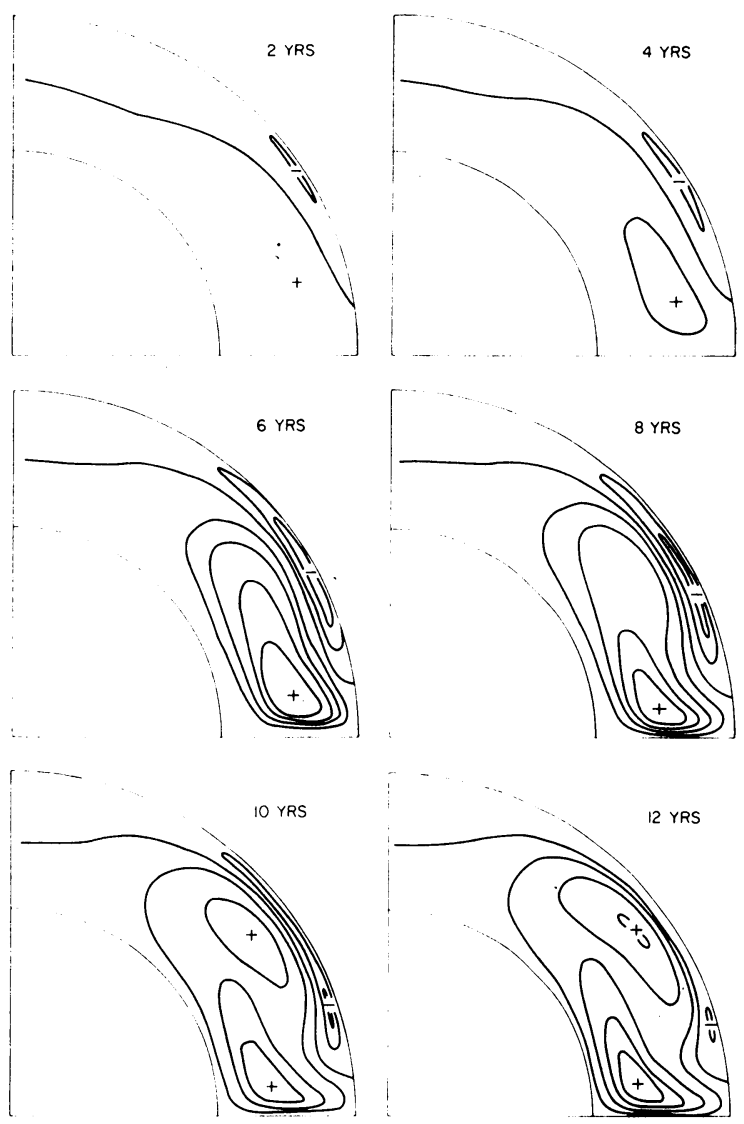

Fig. 2. The equatorial migration of the zone of activity (identified with - sign near the surface) at $2,4,6,8,10$, and $12 \mathrm{yr}$ after the initial state for the axisymmetric incompressible model (Nakagawa and Swarztrauber, 1969).

is generated by a strong distortion of toroidal magnetic field near the surface. The major difference between these two models is that the present model contains the possible mechanism for the formation of sunspots.

It has been shown (Nakagawa and Malville, 1969; Nakagawa, 1970a) that the instability due to the magnetic buoyancy could be identified with the instability arising from the local magnetic shear in a stratified fluid in the proper context of hydromagnetics. In the present model such a magnetic shear can be generated locally due to dispersive nature of oscillations of the interior flow and magnetic field. Recently thermal instability due to ionization has been reported by Defouw (1970), thus this mechanism must be examined in more detail in relation to the hydrogen ionization and hopefully account for Hale's law governing the polarity of emerging sunspots.

In the present study, our attention is directed only to the kinematics of the interior primary magnetic and flow fields, and the observed surface magnetic field is con- 
sidered as the secondary phenomenon. However, we may illustrate the general concept of the ultimate model which incorporates the possible convective phenomena of the Sun. Figure 4 shows such a model. On the surface dominant convective motions are the granulations and supergranulations. The observed rather homogeneous characters of these phenomena all over the solar surface suggest little connection with large scale flow and magnetic fields. Hence we may consider the pertinent physical mechanisms responsible for these phenomena could be confined within the shallow layer of the depth of strong hydrogen ionization zones. Below the strong hydrogen ionization zone

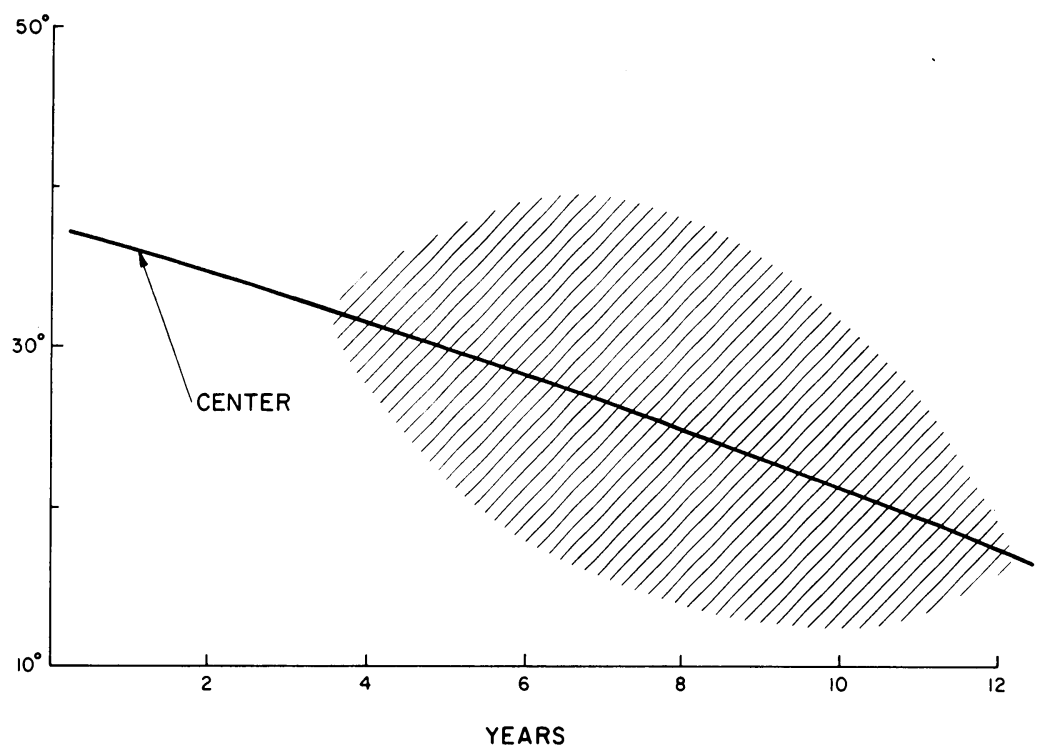

Fig. 3. The 'Butterfly' diagram (Nakagawa and Swarztrauber, 1969). The shaded area represents the strength of the toroidal magnetic field near the surface above $175 \mathrm{G}$.

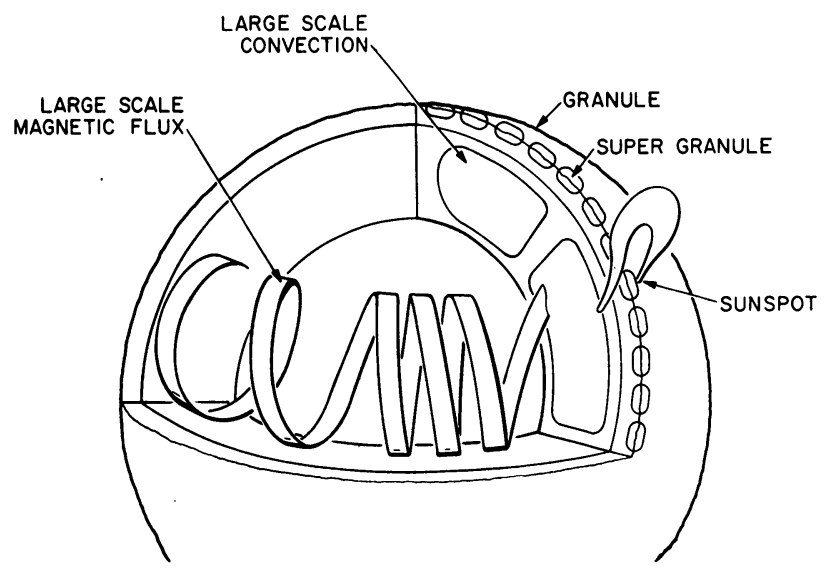

Fig. 4. A schematic model of convective motions and large scale magnetic field of the Sun. 
we may then postulate the existence of large scale convection motion together with the large scale magnetic field for which the present study is directed.

The results of the present study indicate the slow variations of the flow and magnetic field which migrate both in the latitudinal and longitudinal directions. These variations could be identified with the variations of large scale magnetic fields reported by Wilcox (1971). Further, the local strong shear in the flow and magnetic fields could be considered the eruptive zone of sunspots, then the present model does contain this mechanism, although the exact manner of formation of the bipolar sunspots still remains for future study.

\section{Concluding Remarks}

In this paper, models of the solar cycle pertinent to numerical studies of the problem are briefly reviewed. Then the physical characteristics of possible averaged state of axisymmetric equilibria are discussed. Some results of preliminary studies and possible non axisymmetric solutions of the hydromagnetic equations are also presented. It is suggested that the observed surface phenomena are somewhat secondary in nature with respect to the interior solutions. This argument is partially supported by the 'magnetokinematic' model (Leighton, 1969) of the solar cycle which indicates the random nature of the surface phenomena and partially by the Krause model (1967) which emphasizes the induced effect of hydromagnetic turbulence.

It may be worthwhile to note some of the detailed physical characteristics of the solar activity summarized by Babcock (1961) since the large scale model must eventually incorporate these phenomena. The east-west asymmetry and preferred longitude of solar activities could be interpreted in the present non-axisymmetric model in terms of a local concentration of the pitch of the interior magnetic flux. The forward tilt of sunspot axes towards the direction of rotation (Minnaert, 1946) could be interpreted by a faster surface rotation. The present non-axisymmetric model, however, suggests a possible modulation of the surface rotation by the interior flow. In other words, the surface differential rotation could be a function of short-time variations closely correlated with the movements of the large scale surface unipolar magnetic regions.

In summary, it seems apparent that the preliminary task of the hydromagnetic model should be directed to the study of the convective circulation with the magnetic field in the solar convection zone. The one-dimensional model of convection zone such as given by Baker and Temesvary (1966) has to be improved to accommodate some meridional circulations. Currently, a formulation of an axisymmetric model of the solar convection zone with a meridional circulation and poloidal as well as toroidal magnetic field is in progress, and it is hoped that the present incompressible model with simulated convective circulation can be integrated into a more realistic compressible hydromagnetic model in this exceedingly complex problem of great importance.

\section{References}

d'Azambuja, M. and d'Azambuja, L.: 1948, Ann. Obs. Meudon 6, fasc.7.

Babcock, H. D.: 1959, Astrophys. J. 130, 364. 
Babcock, H. W.: 1961, Astrophys. J. 133, 572.

Baker, N. H. and Temesvary, S.: 1966, Tables of Convective Stellar Envelope Models, Institute for Space Studies, NASA, New York.

Busse, F.: 1970, Astrophys. J. 159, 629.

Carrington, R. C.: 1858, Monthly Notices Roy. Astron. Soc. 19, 1.

Chandrasekhar, S.: 1961, Hydrodynamic and Hydromagnetic Stability, Clarendon Press, Oxford, p. 622.

Defouw, R. J.: 1970, Astrophys. J. 161, 55.

Ferraro, V. C. A.: 1937, Monthly Notices Roy. Astron. Soc. $97,458$.

Hale, G. E.: 1908, Astrophys. J. 28, 100, 315.

Hale, G. E.: 1913, Astrophys. J. 38, 27.

Janke, E. and Emde, F.: 1945, Tables of Functions, Dover Publ., New York.

Kato, S.: 1969, Astrophys. J. 157, 827.

Kato, S. and Nakagawa, Y.: 1969, Solar Phys. 10, 476.

Kato, S. and Nakagawa, Y.: 1970, Solar Phys. 14138.

Krause, F.: 1967, Habilitationschrift, University of Jena.

Leighton, R. L.: 1964, Astrophys. J. 140, 1547.

Leighton, R. L.: 1969, Astrophys. J. 156, 1.

Mestel, L.: 1961, Monthly Notices Roy. Astron. Soc. 122, 473.

Minnaert, M.: 1946, Monthly Notices Roy. Astron. Soc. 106, 98.

Nakagawa, Y.: 1970a, Solar Phys. 12, 419.

Nakagawa, Y.: 1970b, Astrophys. Space Sci. 8, 327.

Nakagawa, Y. and Malville, J. M.: 1969, Solar Phys. 9, 102.

Nakagawa, Y. and Swarztrauber, P.: 1969, Astrophys. J. 155, 1111.

Nakagawa, Y. and Trehan, S. K.: 1970, Astrophys. J. 160, 725.

Newton, H. W. and Nunn, M. L.: 1951, Monthly Notices Roy. Astron. Soc. 111, 413.

Spörer, G.: 1894, Publ. Potsdam Obs. 10, Part. I, 144.

Wilcox, J. M.: 1971, this volume, p. 744.

Woltjer, L.: 1959a, Astrophys. J. 130, 405.

Woltjer, L.: 1959b, Astrophys. J. 131, 227.

\section{Discussion}

Meyer: The equations and the boundary conditions of your model are axisymmetric. The solution you showed contains an azimuthal variation. How was the period for this variation determined, was it the outcome of a time evolution or was it introduced by the initial condition?

Nakagawa: The equations are non-axisymmetric and we chose a set of non-axisymmetric initial solutions. The period of variation varies with wave number and at present we are examining different initial conditions to be more general in the future.

Ward: Your model's conversion of kinetic energy, combined with Howard's observations of rapid changes in the velocity fields should constitute at least a partial answer to Altschuler's question earlier this morning. 